\section{Anemia e obesidade: um paradoxo da transição nutricional brasileira}

\author{
Anemia and obesity: a paradox of the nutritional \\ transition in Brazil
}

\author{
1 Instituto Materno Infantil \\ Professor Fernando Figueira, \\ Recife, Brasil. \\ 2 Centro de Pesquisas Aggeu \\ Magalhães, Fundação \\ Oswaldo Cruz, Recife, Brasil. \\ 3 Departamento de Nutrição \\ Universidade Federal de \\ Pernambuco, Recife, Brasil. \\ Correspondência \\ M. Batista Filho \\ Instituto Materno Infantil \\ Professor Fernando Figueira. \\ Rua dos Coelhos 300 \\ Recife, $P E$ \\ 50070-550, Brasil. \\ mbatista@imip.org.br
}

\begin{abstract}
The aim of this study was to analyze the growing prevalence of anemia and overweight/obesity as contrasting trends in Brazil's nutritional transition. Twenty-eight studies published on anemia in children and childbearing-age women were selected, based on statistical representativeness, standardization of laboratory methods, and World Health Organization criteria. Overweight/ obesity in adults was assessed by body mass index: 25 to $29.9 \mathrm{~kg} / \mathrm{m}^{2}$ (overweight) and $\geq 30 \mathrm{~kg} /$ $\mathrm{m}^{2}$ (obesity). Three surveys were compared for analysis of tendencies: 1974/1975 (36.4\%), 1989 (53.5\%), and 2002/2003 (51.9\%) for overweight/ obesity prevalence. In the most representative study on anemia among children $(<11 \mathrm{~g} / \mathrm{dL})$, the prevalence increased from $22.0 \%$ (1974) to $46.9 \%$ (1995/1996). For pregnant woman $(<11 \mathrm{~g} / \mathrm{dL})$, results ranged from 14.7 to $40.4 \%$. Prevalence of anemia among children and overweight/obesity among adults showed similar tendencies over time. Recent evidence of reduction in anemia can be attributed to flour supplementation with iron and folic acid. Anemia and overweight/obesity are associated with significant changes in food intake as a substratum of the nutritional transition.
\end{abstract}

Nutritional Epidemiology; Anemia; Obesity; $\mathrm{Nu}$ tritional Transition
Malaquias Batista Filho 1

Ariani Impieri de Souza 1

Teresa Cristina Miglioli 2

Marcela Carvalho dos Santos 3

\section{Introdução}

Em virtude dos paradigmas habitualmente referenciados, como o enfoque isolado das doenças em sua etiopatogenia, estabeleceu-se a tendência praticamente normativa de se descrever e analisar os eventos do setor saúde e, particularmente, as doenças nutricionais como entidades próprias, autônomas ou, no máximo, aproximadas por "analogias de natureza". Em outras palavras, as deficiências nutricionais formariam agrupamentos de situações conceitualmente associadas, como desnutrição energético-protéica, carências de sais minerais e hipovitaminoses. Ainda assim, a tendência dominante se estabelece no sentido de considerá-las separadamente, de forma autônoma, com sua identidade própria e inconfundível.

Dessa maneira, entre as quase trinta sinonímias utilizadas para designar formas graves de desnutrição energético-protéica, como o kwashiokor, apenas uma, a "síndrome pluricarencial infantil" ou "síndrome pluricarencial hidropigênica” 1 , ressaltava o caráter multicarencial do problema como entidade clínica. No pólo oposto, a associação de problemas relacionados com excessos alimentares e estilos de vida não saudáveis seria bem mais aceitável e usual, como nos casos da obesidade, sobrepeso, dislipidemias e suas múltiplas complicações ou co-morbidades, entre as quais se sobressaem o diabetes mellitus e as doenças cardiovasculares 2,3,4. 
Responsáveis atualmente por $80 \%$ da carga de morbimortalidade dos países ricos e mais da metade nos povos em desenvolvimento 2,5 , as chamadas doenças crônicas não transmissíveis apresentam em comum seus principais fatores biológicos e comportamentais de risco. Entre esses fatores, destacam-se as variáveis nutricionais, representadas pela alimentação hipercalórica e seus desvios específicos: consumo excessivo de açúcares simples, de gorduras animais, de ácidos graxos saturados, de gorduras trans, ao lado do sedentarismo crescente, tabagismo, uso imoderado de bebidas alcoólicas e outras práticas de vida não saudáveis 6,7 .

De forma simplificada, são representações de dois modelos bem distintos e até antagônicos, justificando a conduta de enfoques clínicos e epidemiológicos diferenciados. Entre os dois cenários (doenças carenciais, atingindo sobretudo as crianças, e as doenças crônicas não transmissíveis, predominando amplamente entre adultos e idosos) se delineia o itinerário da transição nutricional, um rápido processo de mudanças nos perfis demográficos e de morbimortalidade bem evidenciado nos últimos cinqüenta anos 8,9,10.

Em princípio, o processo de transição consiste na substituição de um padrão de doenças e mortes característico de um estágio histórico de subdesenvolvimento econômico, social e de saúde por um outro padrão, que resultaria no modelo mais atual dos países de avançada economia de mercado. Os mecanismos internos e externos desse processo e seus resultados, apresentando algumas variantes, teriam em comum esse salto quali e quantitativo no modelo de desenvolvimento econômico-social, implicando necessariamente a mudança de uma situação própria do passado para uma nova e radicalmente diferente situação no presente 11,12. Sobre esse aspecto, provavelmente, não existe nenhum dissenso.

No entanto, a transição nutricional que se desenvolve no Brasil apresenta uma singularidade notável: o agravamento simultâneo de duas situações opostas por definição: uma carência nutricional (a anemia) e uma condição típica dos excessos alimentares, a obesidade ${ }^{13}$. Como entender esse antagonismo de natureza e de tendências num mesmo contexto histórico, num mesmo espaço geográfico e numa mesma população, de forma até independente de sua heterogeneidade sócio-econômica?

É possível que não se tenha uma explicação definitiva ou convincente sobre essa situação conflitante que, inclusive, põe em questionamento o conceito mais literal de transição epidemiológica no campo da nutrição, na medida em que não ocorre a esperada substituição de uma endemia carencial para a elevação de uma outra endemia de natureza oposta - o sobrepeso/obesidade.

Neste artigo, faz-se um exercício inicial de consolidação de resultados que possam representar a coexistência dos dois problemas e uma sistematização de possíveis evidências para a compreensão do que poderia ser um paradoxo epidemiológico.

\section{Procedimentos}

Com esses objetivos, considera-se, de fato, quatro enfoques: (a) um resumo das mudanças nutricionais ocorridas nas últimas três décadas, no Brasil; (b) uma descrição mais detalhada da evolução comparativa do estado nutricional da população adulta (homens e mulheres), com ênfase no binômio sobrepeso/obesidade, incluindo aspectos geográficos e sociais; (c) a consolidação de resultados selecionados sobre a ocorrência de anemia em crianças e mulheres brasileiras, por ordem temporal; em adição, relacionam-se resultados preliminares de recentes pesquisas nos estados de Pernambuco e Paraíba; (d) finalmente, delineiam-se, nas considerações finais, algumas críticas, hipóteses e conclusões que possam ajudar a entender o aparente paradoxo.

Para a inclusão dos resultados referentes à anemia em crianças, foram adotados os seguintes critérios: (a) amostras de base populacional com um mínimo de 400 observações; (b) utilização das técnicas de dosagem da cianometahemoglobina ou uso do Hemocue, na determinação laboratorial da hemoglobina; (c) utilização dos pontos de corte recomendados pelo Comitê de Especialistas da Organização Mundial da Saúde (OMS) para definição de anemia 14. Com estes procedimentos, dos 45 estudos relatados entre 1980 e 2007, apenas 16 foram incluídos, segundo as fontes relacionadas na coluna Estudo de cada tabela. Em relação às mulheres no período reprodutivo, foram adotados os mesmos procedimentos, com uma exceção: aceitação de avaliações de amostras obtidas no pré-natal, tendo em vista a boa representatividade dessas informações em virtude da grande cobertura da assistência obstétrica e o uso rotineiro de exames de sangue neste atendimento.

Considerando os propósitos do estudo, fundamentalmente direcionados na avaliação de mudanças temporais, e levando em conta a semelhança das prevalências entre macrorregiões geográficas, os resultados dos estudos selecionados foram apresentados por ordem cronológica (ano de coleta dos dados primários), de modo a possibilitar uma leitura estatística seqüencial 
da ocorrência de anemias no curso das últimas três décadas.

Excepcionalmente, um dos estudos relatados 15 refere-se ao ano de 1974/1975, fora, portanto, do bloco de informações consolidadas a partir de 1980. Sua inclusão se justifica por se tratar do único trabalho que possibilita uma série temporal de três décadas sucessivas, estando, assim, dentro de uma perspectiva plenamente apropriada para os objetivos do artigo aqui apresentado. Tendo em conta o interesse especial de se dispor de dados produzidos dois anos após a obrigatoriedade de enriquecimento de massas industrializadas com ferro e folato, e não existindo dados publicados a partir de 2006, à exceção do estudo de Pelotas, Rio Grande do Sul, Brasil 16, considerou-se a pertinência e oportunidade de antecipar neste artigo resultados ainda não publicados em veículos de divulgação científica. É o caso das pesquisas relatadas em notas explicativas nas Tabelas 1 e 2 .
Uma vez que o sobrepeso e a obesidade não representariam, ainda, um problema epidemiologicamente significativo em crianças e adolescentes, os resultados referentes a essa condição incluem somente os maiores de vinte anos, segundo sexo. Utilizam-se os resultados de pesquisas de abrangência nacional e aplicam-se, para classificação do estado de nutrição, o índice de massa corporal (IMC), assim calculado: IMC = peso $(\mathrm{kg})$ /altura $\left(\mathrm{m}^{2}\right)$.

$\mathrm{O}$ resultado dessa fórmula foi classificado em quatro categorias: (a) baixo peso: $\mathrm{IMC} \leq 18,5 \mathrm{~kg}$ / $\mathrm{m}^{2}$; (b) peso normal: IMC $>1,85<25,0 \mathrm{~kg} / \mathrm{m}^{2}$; sobrepeso: IMC $\geq 25,0<30 \mathrm{~kg} / \mathrm{m}^{2}$; obesidade: IMC $\geq 30 \mathrm{~kg} / \mathrm{m}^{2}$.

Desde que as fontes dos dados publicados não incluem explicitamente a condição de peso normal (ou eutrofia), a prevalência dessa categoria foi calculada em percentuais, como complemento ou diferença percentual da soma das categorias $(\mathrm{a}+\mathrm{c}+\mathrm{d})-100 \%$, passando, assim, a figurar na representação dos resultados que não constam do artigo original 17.

Prevalência de anemia por localidades e anos de coleta de dados de campo em crianças brasileiras, a partir dos anos 80.

\begin{tabular}{|c|c|c|c|c|c|}
\hline Localidade & Estudo & Ano de coleta & Grupo estudado & Amostra (n) & Anemia (\%) * \\
\hline Paraíba (8 municípios) & Oliveira et al. 33 & 1982 & 6-60 meses & 1.558 & 19,3 \\
\hline São Paulo (município) & Monteiro et al. 15 & $1984 / 1985$ & 6-59 meses & 912 & 35,6 \\
\hline Bahia (semi-árido) & Assis et al. 36 & 1989 & $<6$ anos & 745 & 22,2 \\
\hline Porto Velho (Rondônia) & Cardoso et al. 37 & 1990 & $<5$ anos & 306 & 41,5 \\
\hline Piauí (estado) & Santos 38 & 1991 & 2-4 anos & 742 & 33,8 \\
\hline Paraíba (8 municípios) & Oliveira et al. 33 & 1992 & 6-60 meses & 1.287 & 36,4 \\
\hline São Paulo (município) & Monteiro et al. 15 & 1995/1996 & $0-5$ anos & 1.256 & 46,9 \\
\hline Salvador (Bahia) & Assis et al. 39 & 1996 & $<5$ anos & 603 & 46,4 \\
\hline Pernambuco (estado) & Batista Filho \& Romani 35 & 1997 & $<5$ anos & 780 & 47,7 \\
\hline Sergipe (estado) & Santos 38 & 1998 & 6-60 meses & 720 & 31,4 \\
\hline Nordeste (9 estados) & Pedraza 21 & 1999 & $11-13$ meses & 770 & 82,0 \\
\hline Brasil (5 regiões) & Spinelli et al. 40 & $1998 / 2000$ & 6-12 meses & 2.715 & 64,5 \\
\hline Maceió (Alagoas) & Santos et al. $41 \star \star$ & 2000 & 6-10 anos & 426 & 25,4 \\
\hline Vitória (Espírito Santo) & Almeida et al. $42 \star \star$ & 2000 & 6m-7anos & 760 & 37,3 \\
\hline Acre (2 municípios) & Muniz et al. 43 & 2003 & 6-60 meses & 677 & 30,6 \\
\hline Pelotas (Rio Grande do Sul) & Assunção et al. 44 & 2004 & $0-5$ anos & 534 & 30,2 \\
\hline Gameleira (Pernambuco) & $\star \star \star$ & 2005 & $<5$ anos & 628 & 46,3 \\
\hline São João do Tigre (Paraíba) & 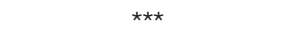 & 2005 & $<5$ anos & 502 & 37,1 \\
\hline Pernambuco (estado) & $\star \star \star$ & 2006 & $<5$ anos & 1.403 & 34,0 \\
\hline
\end{tabular}

* Todos os estudos utilizaram o ponto de corte de 11,0g/dL para caracterizar a anemia;

** $\mathrm{O}$ estudo de Maceió utilizou o ponto de corte de 11,5g/dL; o estudo de Vitória utilizou o ponto de corte de 11g/dL para: 6 -59 meses e 11,5g/dL para 60-84 meses;

*** Fonte: resultados de três pesquisas apresentados em relatório técnico-científico ao Conselho Nacional de Desenvolvimento Científico e Tecnológico - Situação Alimentar, Nutricional e de Saúde no Estado de Pernambuco: Contexto Sócio-econômico e de Serviços; Avaliação da Situação Alimentar e Nutricional e seus Fatores Determinantes em Conglomerados Urbanos e Rurais do Semi-árido do Nordeste e Avaliação da Situação Alimentar e Nutricional e seus Fatores Determinantes em Conglomerados Urbanos e Rurais da Zona da Mata de Pernambuco. 
Tabela 2

Prevalência de anemia em mulheres em idade reprodutiva, segundo alguns trabalhos selecionados e resultados preliminares de pesquisas mais recentes (1980 a 2006).

\begin{tabular}{|c|c|c|c|c|c|}
\hline Localidade & Estudo & Ano de coleta & Grupo estudado & Amostra (n) & Anemia (\%) * \\
\hline São Paulo & Szarfarc 45 & 1977/1981 & Gestantes & 4.539 & 35,1 \\
\hline Estado da Paraíba (3 municípios) & Grillo 46 & 1982 & Gestantes & 484 & 20,0 \\
\hline Rio de Janeiro (pré-natal) & Silva 47 & 1990/1992 & Gestantes & 1.130 & 14,7 \\
\hline Recife (Pernambuco) & World Health Organization 48 & 1990 & Gestantes & 710 & 40,4 \\
\hline Estado do Piauí & Santos 38 & & 14-49 anos & 809 & 26,2 \\
\hline São Paulo & Sinisterra-Rodriguez et al. 49 & 1990 & Gestantes & 691 & 29,2 \\
\hline Recife (Pernambuco) & Arruda 50 & $1992 / 1994$ & Gestantes & 1.007 & 30,9 \\
\hline Estado de Pernambuco & Batista Filho et al. $35 * *$ & 1996 & $10-49$ anos & 1.196 & 24,5 \\
\hline Estado de Pernambuco & 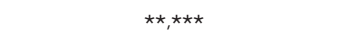 & 2006 & $10-49$ anos & 1.489 & 16,7 \\
\hline Estado de Pernambuco (zona rural) & 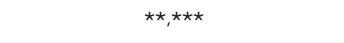 & 2006 & $10-49$ anos & 696 & 15,2 \\
\hline São João do Tigre (Paraíba) & $\star \star \star * \star * \star$ & 2006 & $10-49$ anos & 499 & 17,6 \\
\hline Gameleira (Pernambuco) & 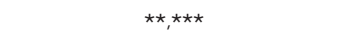 & 2006 & $10-49$ anos & 586 & 31,7 \\
\hline
\end{tabular}

* Todos os estudos utilizaram o ponto de corte de 11,0g/dL para caracterizar a anemia;

** Ponto de corte: $10-11$ anos $<11,5 \mathrm{~g} / \mathrm{dL} ; 12-49$ anos $<12,0 \mathrm{~g} / \mathrm{dL}$;

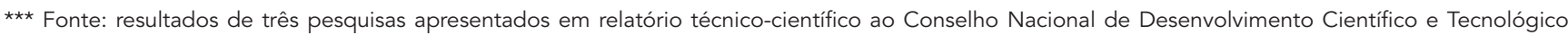
- Situação Alimentar, Nutricional e de Saúde no Estado de Pernambuco: Contexto Sócio-econômico e de Serviços; Avaliação da Situação Alimentar e Nutricional e seus Fatores Determinantes em Conglomerados Urbanos e Rurais do Semi-árido do Nordeste e Avaliação da Situação Alimentar e Nutricional e seus Fatores Determinantes em Conglomerados Urbanos e Rurais da Zona da Mata de Pernambuco.

\section{Três décadas de mudanças}

\section{Resumo do contexto epidemiológico}

A partir de 1974, o panorama nutricional no Brasil apresentou mudanças marcantes e até surpreendentes. Dessa forma, o déficit estatural de crianças menores de cinco anos decaiu em torno de $75 \%$, entre a linha de base de $1974 / 1975$ e os anos de 2002/2003 18. Simultaneamente, iniciase a elevação epidêmica do sobrepeso/obesidade em adultos, com variações notáveis de sexo e de área geográfica. Em sentido oposto à marcante diminuição da desnutrição em crianças, a obesidade em homens adultos praticamente triplicou entre a metade dos anos 70 e o início dos anos 2000, aumentando em mais de $50 \%$ nas mulheres. Em 1974/1975, a prevalência de baixo peso para estatura em mulheres e homens adultos era bem maior do que a ocorrência de obesidade. No entanto, o peso excessivo (IMC entre $25-29,9 \mathrm{~kg}$ / $\mathrm{m}^{2}$ ) já se manifestava em $18 \%$ dos homens e $27 \%$ das mulheres. Desde então, instalou-se o processo epidêmico do sobrepeso/obesidade que, atualmente, já atinge cerca de $50 \%$ da população adulta.

Admite-se, com fundamento em estudos escassos, pontuais e, portanto, pouco conclusivos, que a deficiência de vitamina A está evoluindo para uma situação de controle epidemiológico, já não aparecendo, por exemplo, casos de lesões oculares graves ${ }^{19}$. Acompanhada periodicamente por indicadores clínicos e bioquímicos convencionais e monitorada por medidas de proteção específica (iodatação obrigatória do sal), a deficiência de iodo está controlada há mais de uma década 20

Já a anemia seguiu um curso singular: ao invés de declínio, como as outras deficiências nutricionais, assumiu características epidêmicas, tornando-se, em termos de magnitude, o principal problema carencial do país. Como observação conclusiva desta sinopse, cabe ressaltar as evidências de que tais problemas de natureza conceitualmente oposta (o sobrepeso/obesidade e as anemias) passaram a se comportar nos últimos trinta anos como situações praticamente co-lineares 13 , o que configuraria uma tendência claramente conflitante.

\section{Anemias, um problema em ascensão?}

Duas linhas de observação podem ser seguidas em relação a essa pergunta. A primeira, mais adequada e consistente, seria a construção de séries históricas considerando-se as mesmas populações, nos mesmos territórios, mediante estudos representativos realizados em intervalos regula- 
res de tempos, a fim de possibilitar a representação estatística de tendências. A segunda seria a ordenação temporal de resultados em diferentes populações e diferentes espaços geográficos.

Como se demonstra na Figura 1, nos raros estudos efetuados em amostras representativas das mesmas populações, em tempos diferentes, evidencia-se que a prevalência de anemias tendeu claramente a aumentar nas décadas finais do século passado (menores de cinco anos - São Paulo e Paraíba - e em escolares de Pernambuco), manifestando-se uma possível tendência de redução segundo dados pesquisados após 2005, portanto na década atual. No caso do Município de São Paulo, que expressa o exemplo mais representativo, a prevalência do problema em menores de cinco anos aumentou de $22 \%$ para 46,9\%. Na Paraíba, entre 1982 e 1992, o aumento foi de $19,3 \%$ para $36,4 \%$. Entre escolares do Recife, dois comportamentos distintos: num primeiro momento, a freqüência das anemias se elevou de $8,8 \%$ (1982) para $18,9 \%$ (2001), enquanto na avaliação mais recente (2005) decaiu para $13,4 \%$. Entre menores de cinco anos, em Pernambuco, inquérito de base populacional demonstra uma diminuição de prevalência de 46,7\%, em 1997 , para 34\% em 2006.
Os três estudos selecionados referentes à década de 80 demonstraram prevalências de anemia em crianças com um valor mínimo de 19,3\% e máximo de 35,8\%. Já entre 1990 e 2000, a ocorrência mais baixa foi encontrada em dois municípios do Acre $(30,5 \%)$ e a mais elevada (82\%), uma amostra aleatória de crianças de 1113 meses, sorteada entre trezentos municípios de mais elevada mortalidade infantil do Nordeste brasileiro 21. Por fim, nas pesquisas de campo efetuadas após o ano de 2000, a menor prevalência encontrada foi de 25,4\% (Maceió, Alagoas), contrapondo-se à maior ocorrência verificada, de 46,3\% (Gameleira, Pernambuco), como se pode observar na Tabela 1.

Na Tabela 2, foram consolidadas informações publicadas ou ainda inéditas sobre prevalência de anemia em várias localidades do Brasil. Reúnem-se amostras de gestantes atendidas no pré-natal (seis relatos) ou avaliadas a partir de amostras de base populacional (outros seis relatos), reportando-se, nesta condição, às mães de crianças sorteadas para inquéritos sobre saúde $\mathrm{e}$ nutrição, recentemente concluídos em sua etapa de trabalho de campo e processamento de dados, nos estados da Paraíba e Pernambuco.

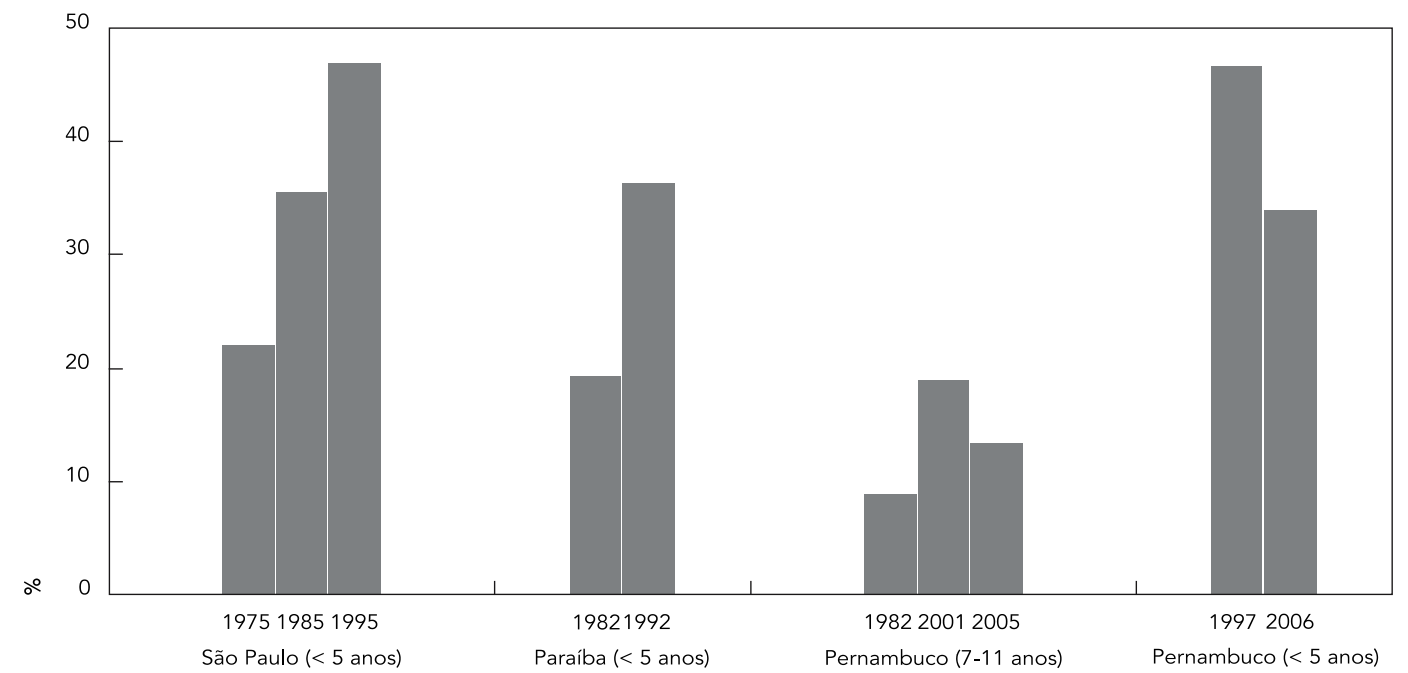

Fonte: Monteiro et al. 15, Oliveira et al. 33, Lemos 34, Batista Filho \& Romani 35 e Situação Alimentar, Nutricional e de Saúde no Estado de Pernambuco: contento sócio-econômico e de serviços (Relatório enviado ao Conselho Nacional de Desenvolvimento Científico e Tecnológico). 
Observa-se, com raras exceções (caso do Rio de Janeiro, em 1990/1992, e de Pernambuco, a partir de 2006), que as prevalências de anemia em gestantes ou em mulheres em idade reprodutiva situavam-se acima de $20 \%$. Os valores mais elevados ocorreram no Recife $(40,4 \%)$ em mulheres examinadas no último trimestre da gravidez, enquanto os mais baixos (15,2\% e 16,7\%) foram encontrados, respectivamente, nos estados da Paraíba e Pernambuco, em estudos mais recentes de mulheres não gestantes.

\section{A pandemia do sobrepeso/obesidade}

Descreve-se, na Figura 2, em homens e mulheres adultas, a evolução do estado nutricional segundo o IMC, entre 1974/ 1975 e 2002/2003. Verificase que o baixo peso declinou e praticamente se estabilizou em níveis aceitáveis a partir de 1989, enquanto a obesidade triplicou em homens, elevando-se de 2,8\% para 8,8\%. Entre as mulheres, a ocorrência de obesidade, que, inicialmente, era três vezes maior que a encontrada nos homens, manteve-se praticamente estável em torno de $13 \%$ nas avaliações efetuadas em 1989 e 2002/2003. No mesmo período, a prevalência de normalidade antropométrica, que era de $71,4 \%$ entre os homens em $1974 / 1975$, caiu para $47,4 \%$ na última avaliação. Entre as mulheres, a prevalência da normalidade antropométrica, segundo o IMC, declinou de $53,4 \%$ para $42,7 \%$. Como se observa, as grandes mudanças da situação nutricional da população adulta, acarretando marcante diminuição da normalidade antropométrica, resultou, fundamentalmente, do aumento do sobrepeso/obesidade.

Demonstra-se, na Tabela 3, o curso temporal das prevalências de obesidade em mulheres do Brasil em três décadas, conforme a renda familiar distribuída em quintis. Observa-se, nos estratos mais pobres (1o e 2o quintis), que o problema mais do que duplicou sua freqüência no primeiro período (1975/1989) e aumentou significativamente no segundo (1989/2003), ao passo que, nas famílias de renda mais elevada (3o, 4o e 5o quintis), a prevalência da obesidade aumentou no primeiro período, tendendo a redução na segunda fase da evolução temporal.

\section{Considerações finais}

Parece surpreendente que, à medida que o desenvolvimento econômico e social amplia consideravelmente o acesso efetivo aos alimentos, informações, serviços, ações de saúde e outros

Figura 2

Classificação antropométrica de homens e mulheres adultos no Brasil, segundo o índice de massa corporal, entre 1974/1975 e 2002/2003.

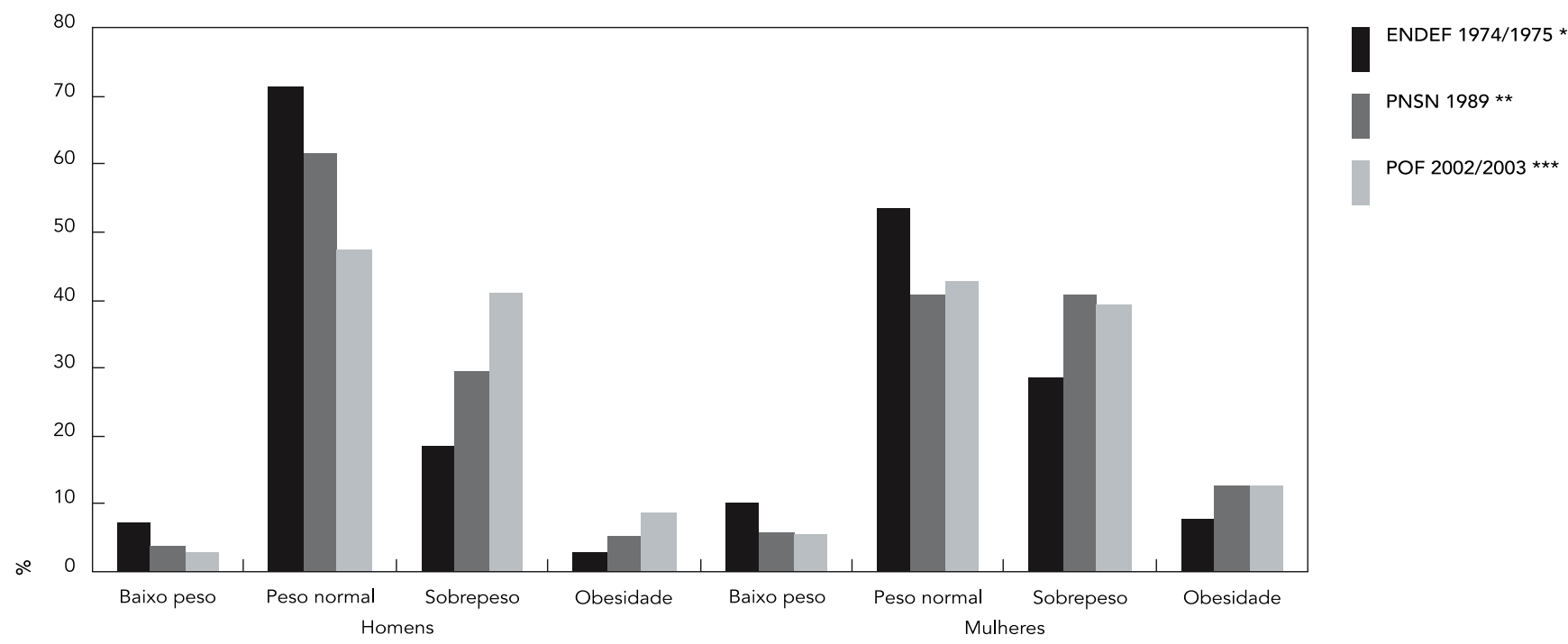

ENDEF: Estudo Nacional da Despesa Familiar; PNSN: Pesquisa Nacional sobre Saúde e Nutrição; POF: Pesquisa de Orçamentos Familiares.

Fonte: Instituto Brasileiro de Geografia e Estatística 17. 
Mudanças na prevalência de obesidade em mulheres adultas, segundo a renda familiar em quintis, no Brasil, em 1975, 1989 e 2003.

\begin{tabular}{|c|c|c|c|c|c|}
\hline \multirow[t]{2}{*}{ Quintis de renda familiar } & \multicolumn{3}{|c|}{ Prevalência de obesidade (\%) } & \multicolumn{2}{|c|}{ Razão de prevalência (IC95\%) } \\
\hline & 1975 & 1989 & 2003 & $1975-1989$ & $1989-2003$ \\
\hline I quintil & 2,6 & 8,9 & 11,2 & $3,27(2,64-4,06)$ & $1,26(1,11-1,43)$ \\
\hline II quintil & 5,7 & 11,7 & 13,5 & $1,97(1,65-2,34)$ & \\
\hline III quintil & 8,8 & 14,8 & 13,5 & $1,65(1,40-1,94)$ & $0,90(0,81-0,99)$ \\
\hline IV quintil & 11,0 & 14,3 & 14,1 & $1,30(1,10-1,52)$ & \\
\hline$V$ quintil & 8,6 & 12,7 & 11,5 & $1,42(1,23-1,65)$ & \\
\hline Total & 7,4 & 12,4 & 13,0 & $1,63(1,47-1,80)$ & $1,03(0,95-1,12)$ \\
\hline
\end{tabular}

Fonte: adaptado de Monteiro et al. 51.

condicionantes que interferem positivamente no estado nutricional, possa observar-se a ocorrência simultânea e crescimento co-linear de dois problemas de natureza oposta, como o sobrepeso/obesidade por um lado e as anemias carenciais por outro. Essa duplicidade de tendências seria absolutamente imprevisível trinta anos atrás, quando começaram a se delinear, em nível epidemiológico, as grandes mudanças no cenário nutricional da população brasileira. Assim, mais do que uma simples análise dos dois problemas isoladamente ou em conjunto, torna-se importante fazer uma reflexão retrospectiva dos enfoques dominantes na avaliação da problemática alimentar e nutricional do Brasil, e mesmo em nível internacional, com evidentes implicações na definição de prioridades e focos de interesse em sua abordagem.

No último século e, particularmente, entre $1950 / 1980$, predominou o que se poderia chamar paradigma das proteínas e calorias como princípio referencial para descrição e análise da situação alimentar e nutricional, com um enfoque claramente centrado na desnutrição energéticoprotéica das crianças 22,23 . Qualidade e quantidade de proteínas e, secundariamente, quantidade de calorias, com uma extensão complementar à questão das vitaminas, era a visão que se impunha aos estudiosos, planejadores e gestores na área de alimentação e nutrição 24 .

Não se tratava, de fato, de uma compreensão própria do Brasil, desde que a mesma perspectiva prevalecia na Food and Agriculture Organization (FAO), na OMS, no Fundo das Nações Unidas para a Infância (UNICEF), no Banco Mundial e nos grandes centros internacionais de estudos e pesquisas. A procura de alimentos ou misturas alimentares de elevado valor biológico (protéico) e baixo custo simboliza bem o pensamento do- minante sobre o problema alimentar/nutricional e os rumos das políticas e das pesquisas para seu encaminhamento. No caso do Brasil, essa perspectiva conceitual foi conflitantemente confrontada com os resultados de um dos maiores e mais completos inquéritos alimentares do mundo, o Estudo Nacional da Despesa Familiar (ENDEF1974/1975) 25, ao se demonstrar que as proteínas, com uma adequação média em torno de $200 \%$ das recomendações, não seriam o fator limitante básico da alimentação da população, mesmo nas famílias de mais baixa renda. A chave explicativa do problema deslocou-se, então, para o déficit calórico 26. De fato, o equívoco, bem observado no caso brasileiro, passou a ser também considerado em termos universais. O artigo O Fiasco das Proteínas pode ser tomado como emblemático da nova percepção do problema alimentar/nutricional 27.

Essa mudança de perspectivas transcorreu no mesmo momento em que se acumulavam, na segunda metade da década de 70 e, principalmente, nos anos 80, evidências consistentes de que a situação nutricional do país estava se transformando rapidamente. Na realidade, o processo de mudança foi mais rápido que sua própria percepção, em nível conceitual, político e epidemiológico. Assim, no inquérito do ENDEF (1974/1975) 25, ao lado da elevada prevalência de desnutrição em crianças, já figurava o problema do sobrepeso/obesidade em maiores de vinte anos, com uma prevalência bem maior que o próprio déficit de peso nos adultos $(21,4 \%$ versus $7,2 \%$ entre os homens e $36,4 \%$ versus $10,2 \%$ entre as mulheres), razão de prevalência três vezes mais elevada. No entanto, tal situação não chegou a ser valorizada nos estudos publicados na época, em virtude da preocupação quase exclusivista com o problema da desnutrição energético protéica. A mesma ob- 
servação poderia justificar o pouco interesse pela situação das anemias e de outros problemas, como a deficiência de vitamina A, de forma que, ainda hoje, os estudos efetivamente confiáveis sobre estas carências específicas são escassos e pouco consistentes, em termos de representatividade amostral, padronização de métodos de avaliação e referencial crítico para assegurar sua validade interna e externa. Muito provavelmente, o aspecto imprevisto dos resultados agora explicitados se relaciona mais com a discordância dos conceitos e atitudes referentes aos problemas nutricionais do que com o paradoxo dos dados comparados. Logo, a questão seria fundamentalmente epistemológica.

Em relação ao sobrepeso/obesidade, o entendimento básico passa necessariamente por um balanço energético excessivamente positivo, configurado no binômio consumo/gasto calórico. No caso do Brasil, os dois termos desta relação têm se alterado rapidamente 7,17,28, com o comprovado aumento do consumo alimentar e a redução progressiva dos gastos calóricos causada pelo crescente sedentarismo da população. Conta-se, atualmente, uma disponibilidade alimentar de mais de três mil calorias para uma necessidade estimada pela FAO em 2 mil calorias per capita/dia 29. Há, assim, um excedente potencial de cerca de $50 \%$ de calorias para cada brasileiro, o que poderia justificar em grande parte a prevalência de mais de $50 \%$ de sobrepeso/obesidade na população adulta.

Relatório recente do Instituto Brasileiro de Geografia e Estatística 17 demonstra as grandes mudanças ocorridas no consumo alimentar das famílias brasileiras em relação ao suprimento de proteínas e calorias e suas fontes. Evidencia-se, ademais, que a desigualdade de condições sócio-econômicas entre regiões e entre estratos de renda não tem o mesmo papel do passado na distribuição geográfica e social da desnutrição/ obesidade, estabelecendo-se uma situação de homogeneidade e, inclusive, de tendência para a diminuição do sobrepeso/obesidade nas faixas de renda familiar mais elevada.

Embora sem se dispor de dados efetivamente consistentes em âmbito nacional, existem evidências convincentes de que o problema das anemias em crianças brasileiras tenha acompanhado o mesmo ritmo da pandemia do sobrepeso/obesidade em adultos 13. Os inquéritos nutricionais realizados em três décadas sucessivas são bem indicativos de que o comportamento temporal das anemias em crianças tem acompanhado as tendências da ocorrência de sobrepeso/obesidade em adultos; em relação às mulheres, as informações analisadas seriam menos conclusivas. Ainda assim, os conjuntos de resultados das décadas de 80 e 90, comparados entre si, são demonstrativos de um aumento de prevalência deste problema.

Ao lado das evidentes limitações descritivas, os estudos sobre fatores causais das anemias têm sido pouco valorizados. Na realidade, apenas dois estudos são demonstrativos do papel das mudanças alimentares no agravamento epidemiológico do problema em crianças menores de cinco anos, em São Paulo e em Pernambuco. O primeiro demonstrou o papel da elevação do consumo de leite fluido no aumento das anemias, por dois prováveis mecanismos: substituição de outras fontes alimentares mais ricas em ferro e bloqueio parcial da absorção intestinal do ferro ${ }^{30}$. O outro estudo, realizado com dados do inquérito alimentar/nutricional no Estado de Pernambuco, chega a conclusões bem semelhantes 31 . Seria um outro paradoxo, pois os conhecimentos até então estabelecidos consideravam o leite um alimento insubstituível na alimentação das crianças, ao passo que, agora, configura-se como o principal causador do problema carencial de maior magnitude na população brasileira: a anemia nas crianças.

Em resumo, o avanço representado pela maior disponibilidade e acesso aos alimentos em nível familiar e, especificamente, o maior consumo do que seria um alimento nobre para as crianças, o leite, acabaram atuando como fatores de risco para mudanças adversas de grande magnitude no processo nutricional. Essas mudanças se desenvolveram quase imperceptivelmente, só sendo explicitadas quando o sobrepeso/obesidade, por um lado, e a anemia, por outro, alcançaram mais da metade da população de adultos e quase a metade da população de crianças. Com uma agravante peculiar: o desinteresse por seu aspecto mais relevante, o substrato alimentar do próprio processo de transição nutricional.

Como questão final, os dados mais recentes parecem indicar que, a partir de 2005, a ocorrência de anemias em crianças, escolares e adultos estaria declinando, segundo alguns resultados apresentados neste artigo, mudando para menos as tendências temporais de aumento do problema. Em princípio, isso poderia ser atribuído ao uso obrigatório de ferro e folato nas massas alimentares de consumo mais generalizado, como os derivados industriais do trigo e do milho 32 . Trata-se, no entanto, de resultados ainda preliminares, necessitando-se de mais estudos para documentar este possível efeito sobre as anemias, em nível populacional. 


\section{Resumo}

Objetiva-se analisar a prevalência crescente das anemias e do sobrepeso/obesidade, como tendências conflitantes da transição nutricional do Brasil. Fez-se uma seleção de 28 trabalhos publicados sobre anemia em crianças e mulheres em idade reprodutiva, considerando representatividade estatística, padronização de técnicas laboratoriais e critérios recomendados pela Organização Mundial da Saúde. O sobrepeso/obesidade em adultos foi avaliado pelo índice de massa corporal (IMC): entre 25 e 29,9kg/m² (sobrepeso) $e \geq$ $30 \mathrm{~kg} / \mathrm{m}^{2}$ (obesidade). Para análise das tendências, foram comparados três inquéritos, 1974/1975 (36,4\%), 1989 (53,5\%) e 2002-2003 (51,9\%), para a prevalência de sobrepeso/obesidade. No estudo mais representativo sobre anemias em crianças $(<11 \mathrm{~g} / \mathrm{dL})$, a prevalência elevou-se de 22\% (1974) para 46,9\% (1995-1996). Nas gestantes $(<11 \mathrm{~g} / \mathrm{dL})$, os resultados variaram entre 14,7\% e 40,4\%. Conclui-se que as prevalências de anemia em crianças e sobrepeso/obesidade em adultos apresentaram tendências semelhantes de evolução temporal. Evidências mais recentes de redução das anemias poderiam ser atribuídas ao enriquecimento das massas alimentares com ferro e folato. As anemias e o sobrepeso/obesidade estariam associados às mudanças no consumo alimentar, como substrato da transição nutricional.

Epidemiologia Nutricional; Anemia; Obesidade; Transição Nutricional

\section{Referências}

1. Batista Filho M, Sigulem DM, Nóbrega FL. Nomenclatura e classificação da desnutrição. Parte 1: desnutrição protéico-calórica. J Pediatr (Rio J) 1976; 41:45-51.

2. World Health Organization. Global strategy on diet, physical activity and health. Food Nutr Bull 2004; 25:292-302.

3. Gomes MB, Giannella-Neto D, Mendonça E, Tambascia MA, Fonseca RM, Réa RR, et al. Prevalência de sobrepeso e obesidade em pacientes com diabetes mellitus do tipo 2 no Brasil: estudo multicêntrico nacional. Arq Bras Endocrinol Metab 2006; 50:136-44.

4. Mariath AB, Grillo LP, Silva RO, Schmitz P, Campos IC, Medina JRP, et al. Obesidade e fatores de risco para o desenvolvimento de doenças crônicas não transmissíveis entre usuários de unidade de alimentação e nutrição. Cad Saúde Pública 2007; 23:897-905.

\section{Colaboradores}

M. Batista Filho contribuiu na concepção do artigo, coordenação do grupo e redação do texto. A. I. Souza e T. C. Miglioli contribuíram na pesquisa bibliográfica e revisão final do texto. M. C. Santos contribuiu na pesquisa bibliográfica.
5. Organização Pan-Americana da Saúde. Doenças crônico-degenerativas e obesidade: estratégia mundial sobre alimentação saudável, atividade física e saúde. Brasília: Organização Pan-Americana da Saúde; 2003.

6. Coordenação Geral de Doenças e Agravos NãoTransmissíveis. Síntese da oficina de vigilância em doenças crônicas não-transmissíveis. Ciênc Saúde Coletiva 2004; 9:957-62.

7. Barreto SM, Pinheiro ARO, Sichieri R, Monteiro CA, Batista Filho M, Schimdt MI, et al. Análise da estratégia global para alimentação, atividade física e saúde, da Organização Mundial da Saúde. Epidemiol Serv Saúde 2005; 14:41-68.

8. Frederiksen H. Feedbacks in economic and demographic transition. Science 1969; 166:837-47.

9. Popkin BM. Nutritional patterns and transition. Popul Dev Rev 1993; 19:138-57. 
10. Barreto ML, Carmo EH. Mudanças em padrões de morbimortalidade: conceitos e métodos. In: Monteiro CA, organizador. Velhos e novos males da saúde no Brasil. 2a Ed. São Paulo: Editora Hucitec; 2000. p. 7-32.

11. Escoda MSQ. Para a crítica da transição nutricional. Ciênc Saúde Coletiva 2002; 7:219-26.

12. Ferreira HS, Florêncio TMTM, Fragoso MAC, Melo FP, Silva TG. Hipertensão, obesidade abdominal e baixa estatura: aspectos da transição nutricional em uma população favelada. Rev Nutr 2005; 18:209-18.

13. Batista Filho M, Rissin A. A transição nutricional no Brasil: tendências regionais e temporais. Cad Saúde Pública 2003; 19 Suppl 1:S181-91.

14. World Health Organization. Iron deficiency anemia: assessment, prevention and control - a guide for programme managers. Geneva: World Health Organization; 2001.

15. Monteiro CA, Szarfarc SC, Mondini L. Tendência secular da anemia na infância na cidade de São Paulo (1984-1996). Rev Saúde Pública 2000; 34(6 Suppl):62-72.

16. Assunção MCF, Santos IS, Barros AJD, Gigante DP, Victora CG. Efeito da fortificação de farinhas com ferro sobre anemia em pré-escolares, Pelotas, RS. Rev Saúde Pública 2007; 41:539-48.

17. Instituto Brasileiro de Geografia e Estatísticas. Pesquisa de orçamentos familiares (POF) 2002-2003. Análise da disponibilidade domiciliar de alimentos e do estado nutricional no Brasil. Rio de Janeiro: Instituto Brasileiro de Geografia e Estatística; 2004.

18. Instituto Brasileiro de Geografia e Estatísticas. Pesquisa de orçamentos familiares no Brasil, 2002/2003. Antropometria e análise do estado nutricional de crianças e adolescentes no Brasil. Rio de Janeiro: Instituto Brasileiro de Geografia e Estatística; 2006.

19. West Jr. KP. Extent of vitamin A deficiency among preschool children and women of reproductive age. J Nutr 2002; 132(9 Suppl):2857S-66.

20. Departamento de Alimentação Básica, Ministério da Saúde. Alimentação e nutrição. http:// dtr2004.saude.gov.br/nutricao/iodo_informacoes. php\#prevencao (acessado em 09/Out/2007).

21. Pedraza DF. Anemia em crianças e o programa de alimentação escolar no contexto da segurança alimentar e nutricional no Brasil [Tese de Doutorado]. Recife: Departamento de Nutrição, Universidade Federal de Pernambuco; 2005.

22. Jelliffe DB, Symonds BE, Jelliffe EF. Childhood malnutrition and the technical development of tropical regions: a note based on a field study in Southern Trinidad. East Afr Med J 1960; 37:405-9.

23. Chaves N. Nutrição básica e aplicada. 2ạ Ed. Rio de Janeiro: Editora Guanabara Koogan; 1985.

24. Berg A. Los malnutridos. Examen de políticas. Washington DC: World Bank; 1981. (Serie sobre la Pobreza y las Necesidades Básicas).

25. Instituto Brasileiro de Geografia e Estatística. Estudo nacional de despesa familiar. Dados preliminares: consumo alimentar; antropometria. v. 1. Rio de Janeiro: Instituto Brasileiro de Geografia e Estatística; 1977.
26. Arruda BKG. Padrões e hábitos alimentares da população brasileira. Brasília: Instituto Nacional de Alimentação e Nutrição; 1981. (Documento Técnico do INAN/MS).

27. McLaren DS. The great protein fiasco. Lancet 1974; 7872:93-6

28. Mondini L, Monteiro CA. Mudanças no padrão de alimentação. In: Monteiro CA, organizadores. Velhos e novos males da saúde no Brasil: a evolução do país e suas doenças. 2a Ed. São Paulo: Editora Hucitec; 2000. p. 79-89.

29. Vasconcellos AB. Ações de nutrição e saúde pública: compromisso para alcançar os objetivos de desenvolvimento do milênio. http://dtr2004.saude. gov.br/nutricao/objetivos_milenio.php (acessado em 10/Out/2007).

30. Levi-Costa RB, Monteiro CA. Consumo de leite de vaca e anemia na infância no Município de São Paulo. Rev Saúde Pública 2004; 38:797-803.

31. Oliveira MAA, Osório MM, Raposo MCF. Concentração de hemoglobina e anemia em crianças no Estado de Pernambuco, Brasil: fatores sócio-econômicos e de consumo alimentar associados. Cad Saúde Pública 2006; 22:2169-78.

32. Agência Nacional de Vigilância Sanitária. Resolução RDC n. ${ }^{\circ} 344$ de 13 de Dezembro de 2002. Regulamento técnico para fortificação das farinhas de trigo e das farinhas de milho com ferro e ácido fólico. Diário Oficial da União; $18 \mathrm{dez}$.

33. Oliveira RS, Diniz AS, Benigna MJC, MirandaSilva SM, Lola MM, Gonçalves MC, et al. Magnitude, distribuição espacial e tendência da anemia em pré-escolares da Paraíba. Rev Saúde Pública 2002; 36:26-32.

34. Lemos MCC. Estado nutricional de crianças, adolescentes e adultos no contexto da transição epidemiológica [Tese de Doutorado]. Recife: Departamento de Nutrição, Universidade Federal de Pernambuco; 2007.

35. Batista Filho M, Romani SAM. Alimentação, nutrição e saúde no Estado de Pernambuco: espacialização e fatores sócio-econômicos. Recife: Instituto Materno-Infantil de Pernambuco; 2002. (Série de Publicações Científicas do Instituto Materno-Infantil de Pernambuco, 7).

36. Assis AMO, Santos LMP, Martins MC, Araújo MPN, Amorim DQ, Morris SS, et al. Distribuição da anemia em pré-escolares do semi-árido da Bahia. Cad Saúde Pública 1997; 13:237-43.

37. Cardoso MA, Ferreira MV, Camargo LMA Szarfac SC. Anemia em população de área endêmica de malária, Rondônia. Rev Saúde Pública 1992; 26:161-6.

38. Santos LMP. Bibliografia sobre deficiência de micronutrientes no Brasil: 1990-2000. Anemia. v. 2. Brasília: Organização Pan-Americana da Saúde; 2002.

39. Assis AMO, Barreto M, Gomes GSS. Prevalência e fatores associados à ocorrência da anemia em préescolares na cidade de Salvador, Bahia. Cad Saúde Pública 2004; 20:1633-41.

40. Spinelli MGN, Marchioni DML, Souza JMP, Souza SB, Szarfac SC. Fatores de risco para anemia em crianças de 6 a 12 meses no Brasil. Rev Panam Salud Pública 2005; 17:84-91. 
41. Santos CD, Santos LMP, Figueiroa JN, Marroquim PMG, Oliveira MAA. Anemia em escolares da primeira série do ensino fundamental da rede pública de Maceió, Alagoas, Brasil. Cad Saúde Pública 2002; 18:1757-63.

42. Almeida APC, Zandonade E, Abrantes MM, Lamounier JA. Deficiência de ferro e anemia em crianças de Vitória, ES. Pediatria (São Paulo) 2004; 26:140-50.

43. Muniz PT, Castro TG, Araújo TS, Nunes NB, SilvaNunes M, Hoffmann EHE. Child health and nutrition in the Western Brazilian Amazon: populationbased surveys in two counties in Acre State. Cad Saúde Pública 2007; 23:1283-93.

44. Assunção MCF, Santos IS, Barros AJD, Gigante DP, Victora CG. Anemia em menores de seis anos: estudo de base populacional em Pelotas, RS. Rev Saúde Pública 2007; 41:328-35.

45. Szarfarc SC. A anemia nutricional entre gestantes atendidas em centros de saúde do Estado de São Paulo (Brasil). Rev Saúde Pública 1985; 19:450-7.

46. Grillo MSLC. Ações integradas de enfermagem e nutrição no controle da desnutrição energético protéica e das carências de ferro e vitamina A no Vale do Piancó - PB. Acompanhamento do estado nutricional de gestantes e nutrizes [Dissertação de Mestrado]. João Pessoa: Departamento de Enfermagem, Universidade Federal da Paraíba; 1985.
47. Silva CVC. Vigilância nutricional de gestantes: análise de um modelo em serviço de atenção primária a saúde - Rio de Janeiro [Dissertação de Mestrado]. Rio de Janeiro: Escola Nacional de Saúde Pública, Fundação Oswaldo Cruz; 1994.

48. World Health Organization. Vitamin and mineral nutrition information system (VMNIS). WHO global database on anemia. http://www.who.int/ vmnis/anaemia/prevalence/anaemia_data sources/en/index.html (acessado em 18/ Jul/2007).

49. Sinisterra-Rodriguez OT, Szarfarc SC, Benicio MHA. Anemia e desnutrição maternas e sua relação com o peso ao nascer. Rev Saúde Pública 1991; 25:193-7.

50. Arruda IKG. Deficiência de ferro, folato e anemia em gestantes atendidas no Instituto Materno Infantil de Pernambuco: magnitude, fatores de risco e implicações nos seus conceptos [Tese de Doutorado]. Recife: Departamento de Nutrição, Universidade Federal de Pernambuco; 1997.

51. Monteiro CA, Conde WL, Popkin BM. Income-specific trends in obesity in Brazil: 1975-2003. Am J Public Health 2007; 97:1808-12.

Recebido em 11/Out/2007

Versão final reapresentada em 30/Jan/2008 Aprovado em 26/Fev/2008 